\title{
Pseudo micro-reentrant activation pattern created by coherent mapping of the right atrial free wall: A case report
}

\author{
Masao Takahashi ${ }^{1}$, Rintaro Hojo ${ }^{1}$, Tomoyuki Arai ${ }^{1}$, Takashi Kimura ${ }^{1}$, and Seiji Fukamizu ${ }^{1}$ \\ ${ }^{1}$ Tokyo Metropolitan Hiroo Hospital
}

September 25, 2021

\begin{abstract}
Coherent mapping with CARTO3 is useful to identify the critical isthmus of scar-related AT. However, it has also a pitfall. We present atrial tachycardia (AT) with pseudo micro-reentrant activation pattern created by coherent mapping on the right atrial free wall. It is possible that a pseudo tachycardia circuit was created due to the algorithm for the reconstruction of coherent mapping. Finally, entrainment mapping led to the identification of correct tachycardia circuit and termination of tachycardia by catheter ablation. When using coherent mapping with CARTO3, it is necessary to understand the pitfall well.
\end{abstract}

\section{Hosted file}

pseudo_microreentry_activation_by_coherent.pdf available at https://authorea.com/users/ 310625/articles/538668-pseudo-micro-reentrant-activation-pattern-created-by-coherentmapping-of-the-right-atrial-free-wall-a-case-report 